place. In these regions $\Delta \lambda$ shows a sharp maximum. A similar phenomenon, in an even more striking form, may be expected in the case of $\Delta \lambda$ of the doublet $K \beta_{1}$, as exactly the same group, which is in a state of reconstruction, gives rise to the lines of the doublet.

\section{N. Seljakow.}

A. Krasnikow.

Physical-Technical Institute of X-rays, Sosnowka 2, Leningrad, U.S.S.R., January $\mathrm{I} 8$.

\section{The Fluorescence of Mercury Vapour.}

IN the course of the study on the band absorption and fluorescence of mercury vapour I have obtained some results, which partly complete but partly contradict former experiments. In the present experiments special care has been taken to maintain the mercury vapour at precisely definite conditions.

Wood (Proc. Roy. Soc., 99, 362, I92I) and Wood and van der Lingen (Astrophys. Journ., 54, I 49, I92 I) have found that the fluorescence of mercury vapour cannot be excited in quiescent vapour, but only in vapour which is being distilled from the liquid metal at a temperature of $150^{\circ} \mathrm{C}$. or more. These authors have arrived at this conclusion as the result of experiments made with several exhausted quartz vessels containing mercury. These vessels were heated and illuminated by an aluminium spark. The fluorescence occurred only in the case when the distillation or a stronger evaporation took place, namely, when a part of the vessel was cooled by an air blast or when the temperature of the entire vessel was rising. If the temperature remained constant for a short time or fell, the effect disappeared altogether.

My first experiments upon this subject were made with a quartz Dewar-flask specially designed for absorption measurements. The flask, carefully cleaned and dried by heating, contained between its walls a certain amount of pure liquid mercury. It was connected with a constantly working mercury-vapour pump. The vessel was heated in an electric oven and the fluorescence excited by a mercury arc lamp or by condensed sparks with electrodes of aluminium, cadmium and zinc. When the temperature of the entire vessel was kept constant at about $\mathrm{I} 55^{\circ} \mathrm{C}$. or higher, green fluorescence limited to the beam of the exciting light was definitely observable. With increasing temperature the fluorescence became stronger. When the temperature fell, the fluorescence got gradually fainter and disappeared only at about $155^{\circ} \mathrm{C}$., which is not in agreement with Wood's experiments referred to above. Moreover, by a local cooling of the wall of the vessel by means of an air blast or water, the intensity of fluorescence diminished with diminishing vapour density, instead of rising as reported by Wood.

These experiments were, however, not considered convincing, since in the vessel connected with the pumps a distillation, weak as it might be, could possibly take place. In order to avoid this difficulty, some experiments with sealed vessels were performed. Two quartz tubes provided with quartz plates at the ends were used successively. Each tube containing a few drops of mercury was heated in an electric furnace closed by quartz windows; the temperature was measured at three different places by means of thermopiles fastened to the walls of the tube. The fluorescence radiation excited by an aluminium spark was observed visually and photographically. The fluorescence spectrum was taken with a quartz as well as a glass spectrograph. The results obtained in these experiments fully confirm previous ones. The intensity of fluorescence remains unchanged if the entire tube is kept at constant temperature, i.e. when distillation and stronger evaporation-as by rising temperature-are avoided. No increase of the fluorescent radiation results from local cooling of the vessel.

The total intensity of the visible fluorescence in saturated mercury vapour as well as that of different bands and lines in the visible and ultra-violet part of the spectrum seems to be, for a given exciting light, a function of the temperature only.

In the spectrum of the fluorescence light of mercury vapour excited by an aluminium spark, besides the bands and lines observed by Wood and van der Lingen (loc. cit.), the line $4358 \AA$.U. was found. This line is well seen on the continuous ground of the visible band. It appears at about $200^{\circ} \mathrm{C}$. as a very faint line, gets stronger with rising temperature, and reaches the maximum at $230^{\circ} \mathrm{C}-270^{\circ} \mathrm{C}$., slowly declining at still higher temperatures; at $345^{\circ} \mathrm{C}$. it is scarcely seen. In connexion with the line $435^{8} \AA$.U. $\left(2 p_{2}-2 s\right)$ the behaviour of the line $2537 \AA$.U. (I $\left.S-2 p_{2}\right)$ in the mercury fluorescence spectrum excited by an aluminium spark may be worth mentioning. The latter appears at about $130^{\circ} \mathrm{C}$., its intensity grows with the temperature, reaches a maximum value at about $230^{\circ} \mathrm{C}$. and then rapidly decreases and vanishes at about $260^{\circ} \mathrm{C}$.

Power (Phys. Rev., 26, 761, 1925) and Kapuściński (NATURE, I I6, 863, I925) have observed in the fluorescence spectrum of cadmium vapour excited by some sparks, different from those of cadmium, the line $2 p_{2}-2 s$ analogous to the mercury line $435^{8} \AA$.U. They have observed also two other lines $2 p_{1}-2 s$ and $2 p_{3}-2 s$. No trace of the corresponding mercury lines $546 \mathrm{r} \AA . \mathrm{U} .\left(2 p_{1}-2 s\right)$ and $4047 \AA . \mathrm{U} .\left(2 p_{3}-2 s\right)$ could be found in these experiments.

A detailed account of these experiments will be published elsewhere.

Physical Laboratory

Stefan Batory University, Wilno, Poland, March Io.

\section{Alternating Intensities in Band Lines.}

BANDS in the spectra of neutral and ionised nitrogen show unexplained alternating intensities, and the bands of helium are best interpreted if one assumes that only half the lines are observed, the alternate lines being not merely weak but missing altogether (Mecke, Zeits. f. Phys. 26, 227, I925; 31, 709, 1925). A similar effect seems to be present in oxygen, although the interpretation is less certain. All these molecules are symmetrical, composed of two like atoms. I wish to suggest that the alternations may be connected with the well-known difficulty of assigning the proper period in quantising the rotation of a symmetrical system (Ehrenfest and Tolman, Phys. Rev. 24, 287, r924).

If a dumbbell rotates about the perpendicular bisector of its axis, it returns after half a revolution to a state which cannot be told from the initial state; the place of each end of the dumbbell is taken by the other end. If we wished to quantise the rotation, we might think that the right period for integrating $p d q$ was this half rotation. Then, using $p_{\phi}$, the angular momentum, and $\phi$, the azimuth, we should have $\int_{0}^{\pi} p_{\phi} d \phi=n h, p_{\phi}=n h / \pi$. That is, the angular momentum of successive allowed states would differ by $h / \pi$, twice the normal amount of $h / 2 \pi$, and we

NO. 2946, VOL. I I 7 ] 